\title{
La accesibilidad de las autovías y la teoría de localización industrial
}

\section{The accessibility by the road transport infrastructure and industrial location theory}

\author{
GERARDo RÍOS-QUEZADA* \\ SAÚl ANTONIO OBREGÓN-BIOSCA**
}

\begin{abstract}
The road transport infrastructures allow territorial transformation and encourage the economic and social development. This research considers the Random Utility Theory (RUT) to explore the characteristics that influence the industrial location by generalized linear models. The empirical analysis considers the Mexican Bajio Region, to analyze the effect that induces travel time in the accessibility potential traditionally used in the industrial location. The results shown that this variable should be considered directly proportional to travel time and inversely proportional to the market size, to be accordant to the RUT. The accessibility shows a significant impact but not decisive by itself.
\end{abstract}

Keywords: industrial location, accessibility, regional development, random utility theory.

\section{Resumen}

Las infraestructuras de transporte carretero son elementos vertebradores del territorio e influyen en el desarrollo socioeconómico. Esta investigación considera los supuestos que establece la Teoría de Utilidad Aleatoria (TUA) para describir las características que influyen en la localización manufacturera mediante el empleo de modelos lineales generalizados. El análisis empírico considera la Región Bajío de México. Los resultados muestran que el potencial de accesibilidad debe considerarse directamente proporcional al tiempo de viaje e inversamente proporcional al tamańo del mercado para que sea acorde a la TUA. Además, dicha accesibilidad induce un impacto significativo pero no determinante por sí mismo.

Palabras clave: localización manufacturera, accesibilidad, desarrollo regional, teoría de utilidad aleatoria.

*Instituto Mexicano del Transporte, correo-e: grios@imt.mx

**Universidad Autónoma de Querétaro, correo-e: saul.obregon@uaq.mx 


\section{Introducción}

Las infraestructuras de transporte viario son elementos clave para la transformación territorial y socioeconómica de un país (Obregón-Biosca, 2010; Obregón-Biosca y Junyent-Comas, 2011) y una de sus características es su influencia en la localización de empresas manufactureras (Obregón-Biosca et al., 2014). La presente investigación se enfoca en analizar el efecto que induce la infraestructura de transporte en el fenómeno de la localización industrial; considera como caso de estudio la región centro occidente de la república mexicana debido a la evidencia que muestra la región en la actividad manufacturera durante la última década (Unger et al., 2013).

La presente investigación considera las herramientas y modelos empleados en Figueiredo et al. (2002), Holl (2004a; 2004b), Alañón (2006) y Alañón y Arauzo (2008), y estima las variables que presentan significación en el fenómeno de localización en la Región Bajío (RB) con enfoque en el análisis de la infraestructura del transporte como factor del emplazamiento manufacturero; su formulación se realiza siguiendo la Teoría de Utilidad Aleatoria (TUA) de Mc Fadden (1974), con base en los resultados observados en el caso de estudio.

Con base en la teoría de la utilidad aleatoria se considera la hipótesis: la accesibilidad inducida por las autovías en el mercado regional hacia el nacional y los puertos de importación-exportación ¿̇son los elementos con mayor significación en la localización manufacturera de la RB en comparación al resto de variables que aproximan a explicar el emplazamiento industrial?

Para dar respuesta a dicha interrogante se plantean tres objetivos particulares: 1. Analizar la significación que presenta la accesibilidad intermunicipal en la localización manufacturera de la RB. 2. Determinar qué características municipales inducen efectos positivos y negativos en la instalación de las empresas en la RB. 3. Analizar el efecto que induce el tiempo de viaje en el potencial de accesibilidad propuesto por Harris (1954) frente a la TUA.

\section{Estado del arte}

Los primeros intentos por describir el fenómeno de la localización industrial parten de los modelos de Von Thünen (1826), en los cuales interviene el costo del transporte, la concentración de la actividad económica de Marshall (1920), los puntos de consumo y el suministro de materias primas de Weber (1929) y la metodología de Christaller (1933) y Lösch (1940) sobre el equilibrio general. Teorías más recientes definen un mo- 
delo básico denominado Nueva Geografía Económica (NGE), que se fundamenta en el potencial del mercado, la causalidad acumulativa y la teoría de los lugares centrales determinados por Chistaller-Lösch (Fujita et al., 1999).

El potencial del mercado describe la instalación de una unidad productiva que depende del acceso a los mercados, éste es estimado, para las unidades consideradas, a partir de la suma ponderada de su poder adquisitivo e inversamente proporcional a la distancia (Harris, 1954). Así, la concentración geográfica se desarrolla a partir de la interacción de rendimientos crecientes, los costos de transporte y la demanda.

La empresa elige una localización donde logre disminuir los costos de transporte; prefiere una ubicación cercana a un mercado importante y progresivo (Krugman, 1998), con ello busca asegurar el desarrollo económico. Tal como se menciona en Fujita y Thisse (1996), el equilibrio espacial es un escenario en el que ninguna empresa se vea estimulada a cambiar de localización, ya que si una empresa se encontrase en una situación con desventaja, implicaría la busqueda de un nuevo emplazamiento que le permitiera obtener mayores beneficios.

Existen factores que influyen en los patrones de localización, tal como la mano de obra disponible y su costo; las oportunidades de mercado, los impuestos y subsidios, la infraestructura, accesibilidad al transporte, el espacio, las comodidades, e incluso las decisiones personales (Bannister y Berechman, 2001; Beckmann, 1999; Small, 1982), así como la competencia entre empresas (Hoover y Giarratani, 1971). En Chasco-Yrigoyen y López-García (2010) se expone que la ubicación de las industrias se ve influenciada por factores geográficos y de aglomeración, este último es un factor resultado de las economías de escala, es decir, el beneficio de algunas empresas mejora cuando operan en el contexto de una economía local más grande y toman ventaja de la cercanía de empresas similares (Cohen y Paul, 2005; Johansson y Quigley, 2004). Recientemente los elementos de economías de aglomeración y la existencia de un mercado especializado de trabajo se retoman por la llamada nueva geografía económica (Krugman, 1998).

\subsection{La nueva geografía económica}

La nueva geografía económica (NGE) indica que los beneficios del comercio pueden derivar en una concentración convergente o divergente, lo anterior responde a las características regionales de cada entidad y son explicadas por los costos de transporte, las fuerzas de aglomeración y la dispersión de la actividad económica (Sánchez-Reaza, 2010). La NGE fundamenta su teoría en los estudios de potencial de mercado, la causa- 
lidad acumulativa y la teoría de los lugares centrales. Como se mencionó anteriormente, en Fujita y Thisse (1996) se conceptualiza el equilibrio espacial como un escenario en que ninguna empresa se ve estimulada a cambiar su localización porque implicaría buscar un emplazamiento distinto para obtener mayores beneficios, entonces, las fuerzas de aglomeración y de dispersión (centrípetas y centrífugas) ofrecen las pautas para la localización siempre y cuando se genere un equilibrio general homogéneo.

En Fujita et al. (1999) se expone que dentro de las fuerzas centrípetas se destacan las economías de escala, los costos de transporte, efectos en el tamaño del mercado, acceso a los mercados y productos; todo lo anterior, enlazado a una localización central o estratégica. Las fuerzas centrífugas se relacionan con las deseconomías internas como la congestión y contaminación, alto costo de la renta del suelo, dispersión de los insumos, competencia por los precios y el costo de la mano de obra. En MendozaCota y Pérez-Cruz (2007) se precisa cada una de las dos fuerzas.

$\mathrm{Al}$ partir de que la mejora de la infraestructura del transporte funge como el medio por el cual se logra integrar el mercado, permitiendo mejores conexiones sobre las áreas de menor actividad económica, se establece que la mejora en la infraestructura del transporte desencadena una atracción para la localización industrial (Holl 2004a; 2004b), mientras que en Alañón y Arauzo (2008) se concluye que reducir el tiempo de acceso induce efectos positivos en la instalación.

\subsection{El modelo empírico}

En la literatura se indica que la localización industrial no es un proceso aleatorio sino más bien resultado de una decisión basada en la maximización de beneficios para la empresa. Así, la decisión se toma mediante un análisis de beneficios actuales y futuros que la empresa espera obtener en dicho lugar. Los beneficios locales se determinan por la cercanía de la oferta a la demanda, en otras palabras, por la accesibilidad que posee el centro de producción hacia el mercado, factores de calidad y mejoras en la infraestructura de transporte, localización, costo, demanda y economías de aglomeración. Se observa que un camino para maximizar los beneficios es reducir los costos de transporte -en distancia o tiempo de viaje- lo cual permite modificar la cualidad de accesibilidad. En un sector económico y un municipio característico $j$ los beneficios esperados por una empresa se pueden representar mediante la ecuación 1 propuesta en McFadden (1974). 


$$
\pi_{j}=\sum_{r \in P} \sum_{r \in M_{r}} P_{j k}\left(T_{j k}^{D}\right) q_{j k}-c_{j}\left(w_{j}, g_{j}\left(T_{j k}^{S}\right), q_{j}\right)-f_{j}
$$

donde $P$ es el conjunto de índices regionales, $M_{r}$ el conjunto de índices municipales en la región $r ; q_{j k}$ el tamaño del mercado, $P_{j k}\left(T_{j k}{ }^{D}\right)$ los ingresos medios, $T_{j k}{ }^{D}$ el costo de transporte entre el punto $j$ y el punto $k, c_{j}$ los costos variables de producción, $w_{j}$ el precio de los factores primarios, $g_{j}\left(T_{j k}^{S}\right)$ el costo promedio de los insumos intermedios, $q_{j}$ el costo de venta, $T_{j k}^{S}$ los costos de transporte de entrada, y $f_{j}$ los costos fijos a nivel de empresa. A partir de lo anterior se establece que una empresa se localizará en una región en la que acumule el mayor número de beneficios. El total de nuevas empresas instaladas en un municipio se determina por la intersección de la demanda y las curvas de oferta sobre los beneficios locales esperados por la empresa y el número de nuevos espacios centrales (ecuación 2).

$$
n_{i j t}=f_{i}\left(x_{j t}\right)+\varepsilon_{i j t}
$$

donde: $n_{i j t}$ es el número de empresas instaladas del sector $i$ localizada en el municipio $j, x_{j t}$ el vector de características municipales que afectan los beneficios esperados, y $\varepsilon_{i j t}$ el error aleatorio.

De manera conceptual, se describe que para la perspectiva de la TUA la empresa buscará el sitio para decidir la ubicación que le permita maximizar sus beneficios. Se representa la probabilidad de que la empresa $i$ decida localizarse en la región $j$ en términos de una variable aleatoria discreta, y que caracterice el resultado de esta elección. En Guimarães et al. (2003) se afirma que la variable para conformar los modelos de elección discreta presenta una distribución de Poisson o una distribución semejante, demostrando que los coeficientes del modelo logit condicional se estiman mediante una regresión de un modelo lineal generalizado que adopte una distribución de Poisson, es decir, ambos modelos resultan consistentes para estimar las variables que intervienen en la localización manufacturera. Por lo anterior, la probabilidad de que la región $j$ reciba un número $n_{i j}$ de nuevos establecimientos pertenecientes al subsector $i$ se expresa por la ecuación 3 .

$$
\operatorname{prob}\left(n_{i j}\right)=\frac{e^{-\lambda_{i j} \lambda_{i j}^{n_{i j}}}}{n_{i j !} !} \quad \lambda>0, n_{i t}=0,1,2, \ldots, n
$$


donde $n_{i j}$ es la probabilidad de que en un municipio $j$ se instalen nuevas empresas pertenecientes al subsector $i$, y $\lambda_{i j}$ el parámetro de Poisson de la media sobre el número de empresas instaladas en el municipio $j$ pertenecientes al subsector $i$.

En Guimarães et al. (2003) se indica que el incrementar el número de alternativas de localización en el modelo condicional logit, para el modelo de regresión de Poisson, significa incrementar el número de observaciones. Sin embargo, se necesita plantear que si la decisión de localización toma lugar dentro de un conjunto de zonas restringidas, el análisis deberá considerar la existencia de factores no observables (i.e. las características del conjunto de empresarios), ya que ello responderá a alternativas irrelevantes. En Holl (2004a) se recomienda considerar variables que afectan directamente a la decisión de localización como las variables geográficas y climáticas, comodidades locales que no pueden ser observadas, difíciles de medir o, simplemente, la inexistencia de información.

\section{Metodología}

En coincidencia con lo que se establece en Alañón (2002), el estudio de la localización industrial se debe abordar desde un punto de vista teórico considerando una postura ecléctica y conciliando las aportaciones de las escuelas que permiten el estudio de localización industrial. Se considera el fenómeno como un proceso complejo, ya que las tres escuelas -la neoclásica, de la conducta e institucional (Hayter, 1997)- aportan argumentos válidos y que resultan ser complementarios. La escuela neoclásica centra su análisis en el comportamiento racional; la escuela de la conducta concede una gran relevancia a los factores personales, trata las decisiones de localización como producto de la incertidumbre y la escuela institucional considera implicaciones sociales, no pretende crear modelos abstractos, por lo cual incluye la participación de los clientes y proveedores, sindicatos, sistemas regionales, los gobiernos o la actuación del resto de las empresas.

No obstante, la escuela neoclásica establece que la teoría de localización debe ser analizada partiendo de variables abstractas, es decir, de una estructura deductiva que permita determinar el lugar de emplazamiento industrial, pues resulta ambiguo construir los indicadores característicos de las escuelas de conducta e institucional. Por ejemplo, la complejidad que representa incluir los parámetros que describan los factores personales o la incertidumbre de la empresa para estimar modelos abstractos agregados geográficamente a nivel municipal. 
El proceso metodológico se define en cinco apartados que se detallan a continuación: dado que el análisis de la accesibilidad funge como principal objetivo, se realiza una descripción con mayor detalle sobre las variables espaciales respecto a las variables económicas y sociales; además del análisis de las variables que determinan el acceso a la demanda de los mercados, se discute la propuesta del potencial de la demanda propuesto en Harris (1954), para posteriormente analizar el efecto que induce al ser integrado en el modelo econométrico basado en la TUA.

\subsection{La zona de estudio}

Los primeros análisis de la RB se reportan en Bassols-Batalla (1979; 1993), donde se definía como una zona delimitada a un nivel de agregación tal que no permitía identificar a los municipios que la conformaban y tendía a ser territorial más que económica. Recientemente, en sentido opuesto, Rodríguez-González y Caldera-Ortega (2013) identifican el nivel de desarrollo de la RB por medio de un análisis inter e intramunicipal, evaluando los indicadores de medición de desarrollo local (Índice de Desarrollo Humano). Lo anterior, con el propósito de volver útiles dichos indicadores para optimizar las herramientas de diseño y evaluación de políticas públicas de los gobiernos locales que pretendan establecer una estrategia de desarrollo regional.

En congruencia con esta investigación, en Unger et al. (2013) se caracteriza a la competitividad económica de los municipios que integran la RB; se destacan las actividades principales en función del producto interno bruto y su grado de integración, además, se delimita en función de la accesibilidad, tiempos de desplazamiento e intensidad de interacciones. En el análisis se incluyen 61 municipios pertenecientes a seis entidades federativas, ordenados en seis corredores carreteros. Destaca la presencia de mayor actividad manufacturera en la RB sobre el resto de actividades económicas y acentúa el corredor Celaya-Querétaro, Aguascalientes y San Luis Potosí, con la mayor propensión a dicha actividad.

En la información expuesta en Unger et al. (2013) los municipios de la RB que presentan mayor actividad manufacturera se conectan al corredor troncal de la red carretera federal, definido por la Secretaría de Comunicaciones y Transportes como el corredor Querétaro-Irapuato-LeónLagos de Moreno-Aguascalientes-Zacatecas-Torreón-Chihuahua-Cd. Juárez. Así, el presente trabajo se basa en lo expuesto por Unger et al. (2013) para definir los municipios que integrarán la RB, asimismo se considera: a) Incluir los municipios que conforman las zonas metropolitanas (ZM) localizadas en la RB (Sedesol-Conapo-Inegi, 2012), b) Bajo un criterio similar al utilizado en Holl (2004a), considerar los municipios 
que tienen población mayor a los 10 mil habitantes. En este trabajo se incluyen los municipios que poseen población mayor a 50 mil habitantes, que no forman parte de las ZM pero tienen acceso al corredor troncal Querétaro-Cd. Juárez (cuadro 2).

Y con referencia a los trabajos de Figueiredo et al. (2002), Holl (2004a; 2004b), Alañón (2006) Alañón y Arauzo (2008) se define el municipio como la unidad geográfica de análisis. En el mapa 1 se muestran los municipios considerados que integran la zona de estudio, mientras que en el cuadro 1 se muestra la descripción y relación entre las variables empleadas en los modelos, en el cuadro 2 se presenta el resumen de estadísticos.

\section{Mapa 1 \\ El ámbito de estudio}

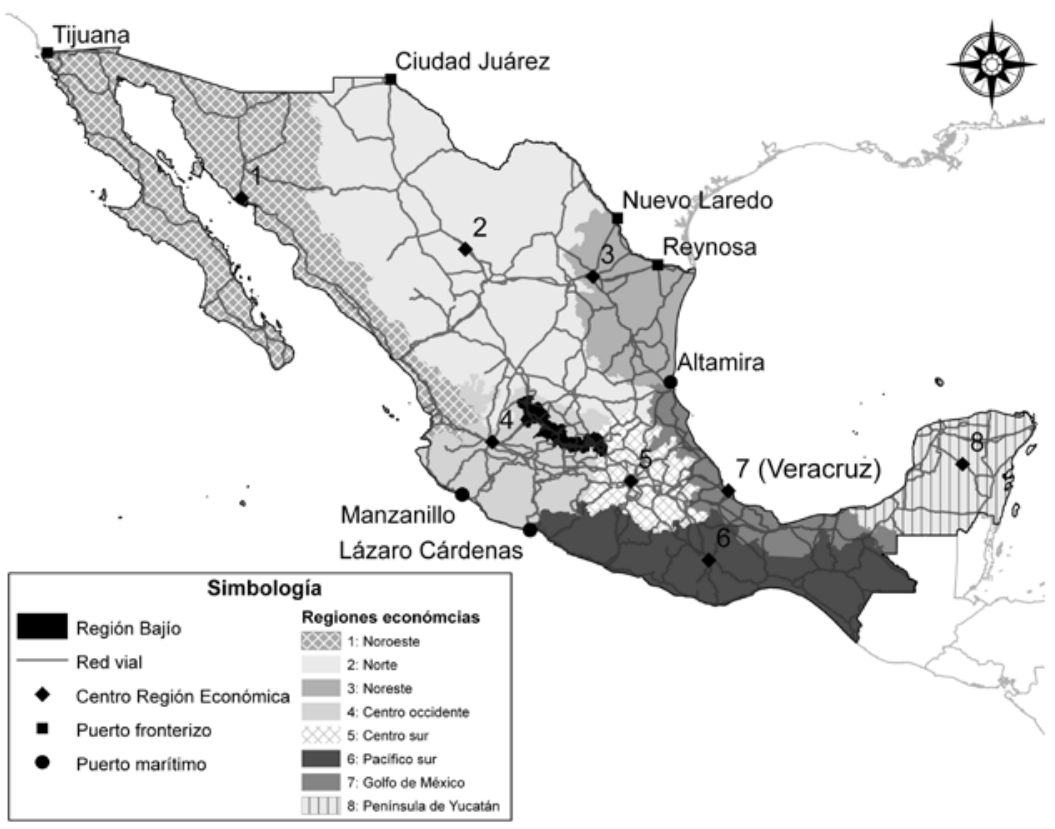

Fuente: elaboración propia. 


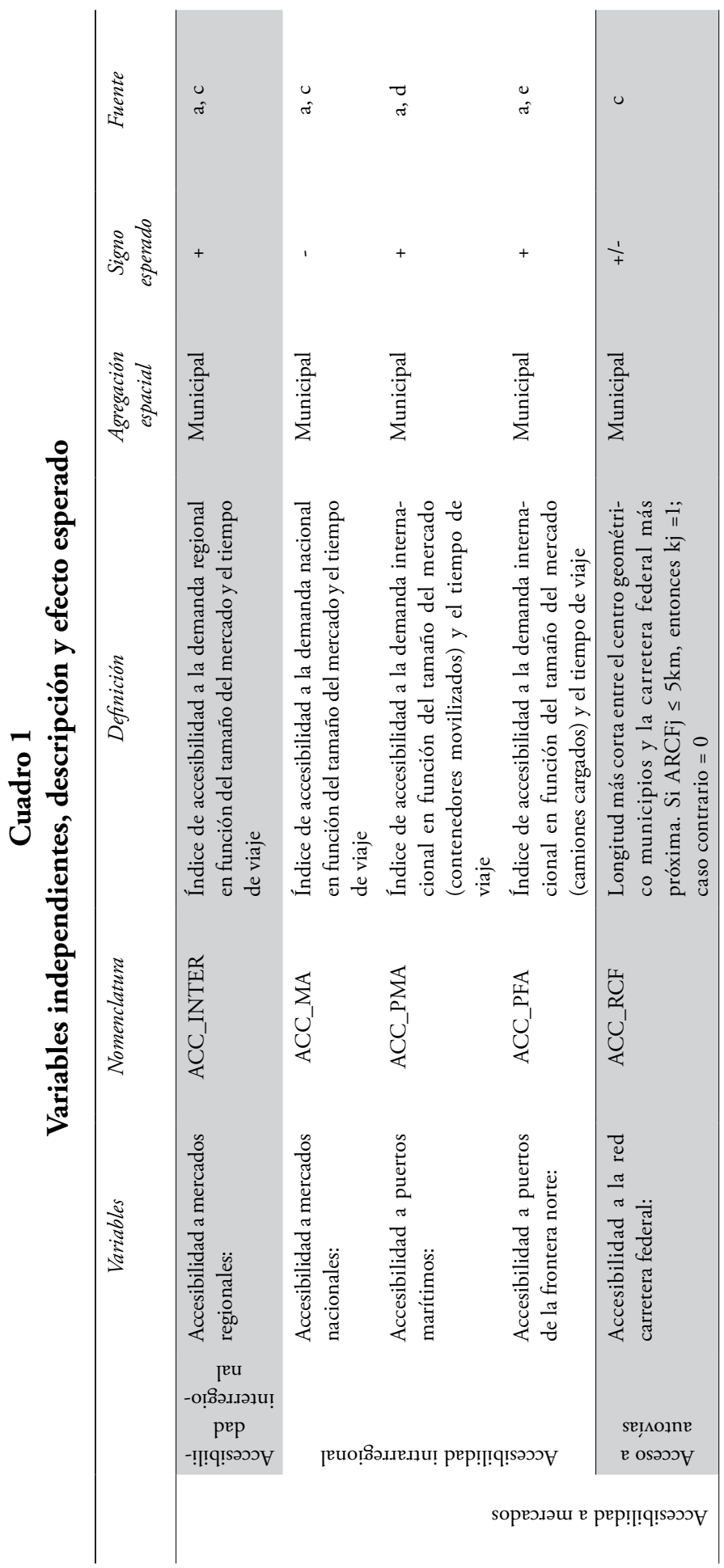




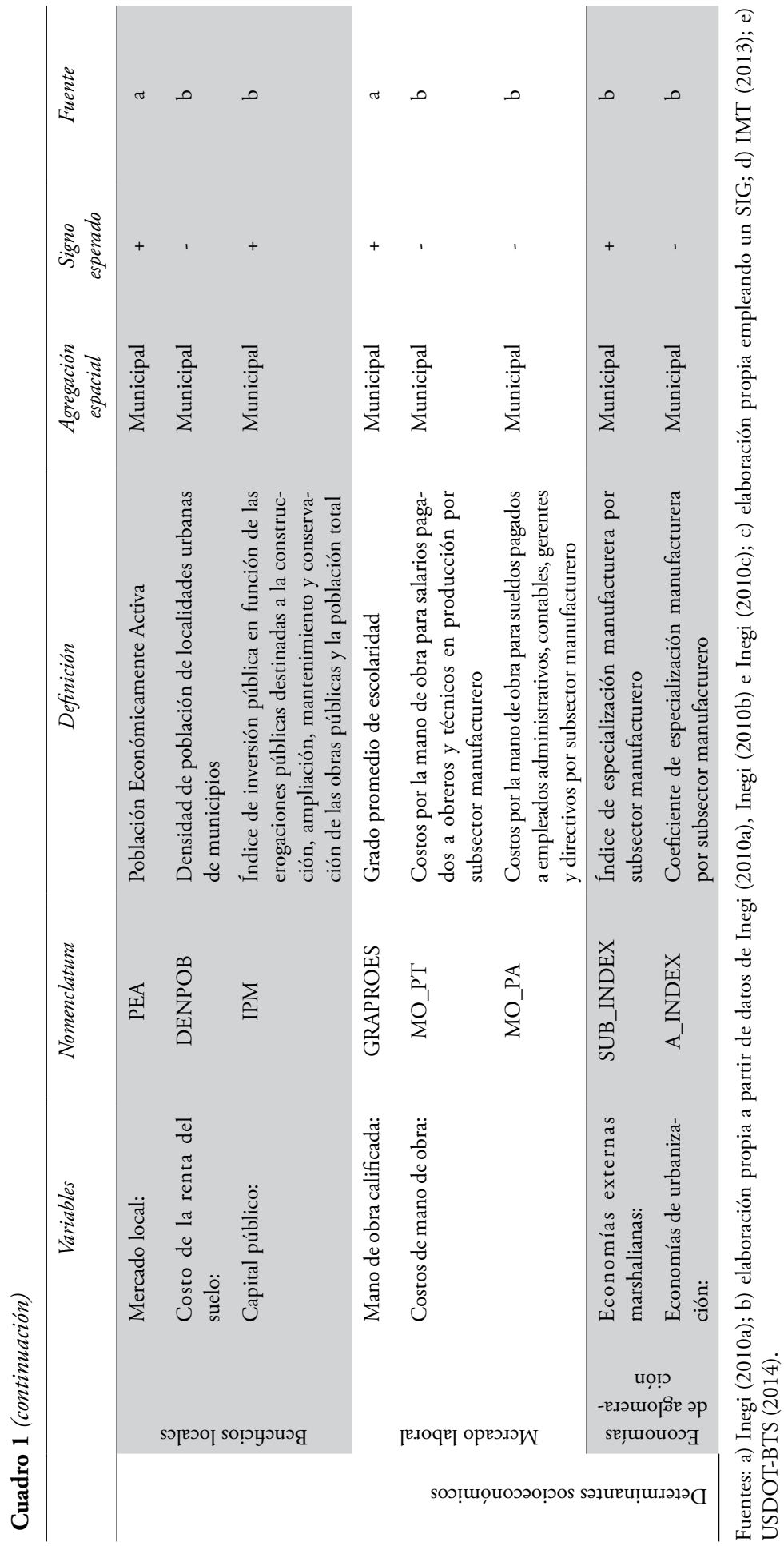




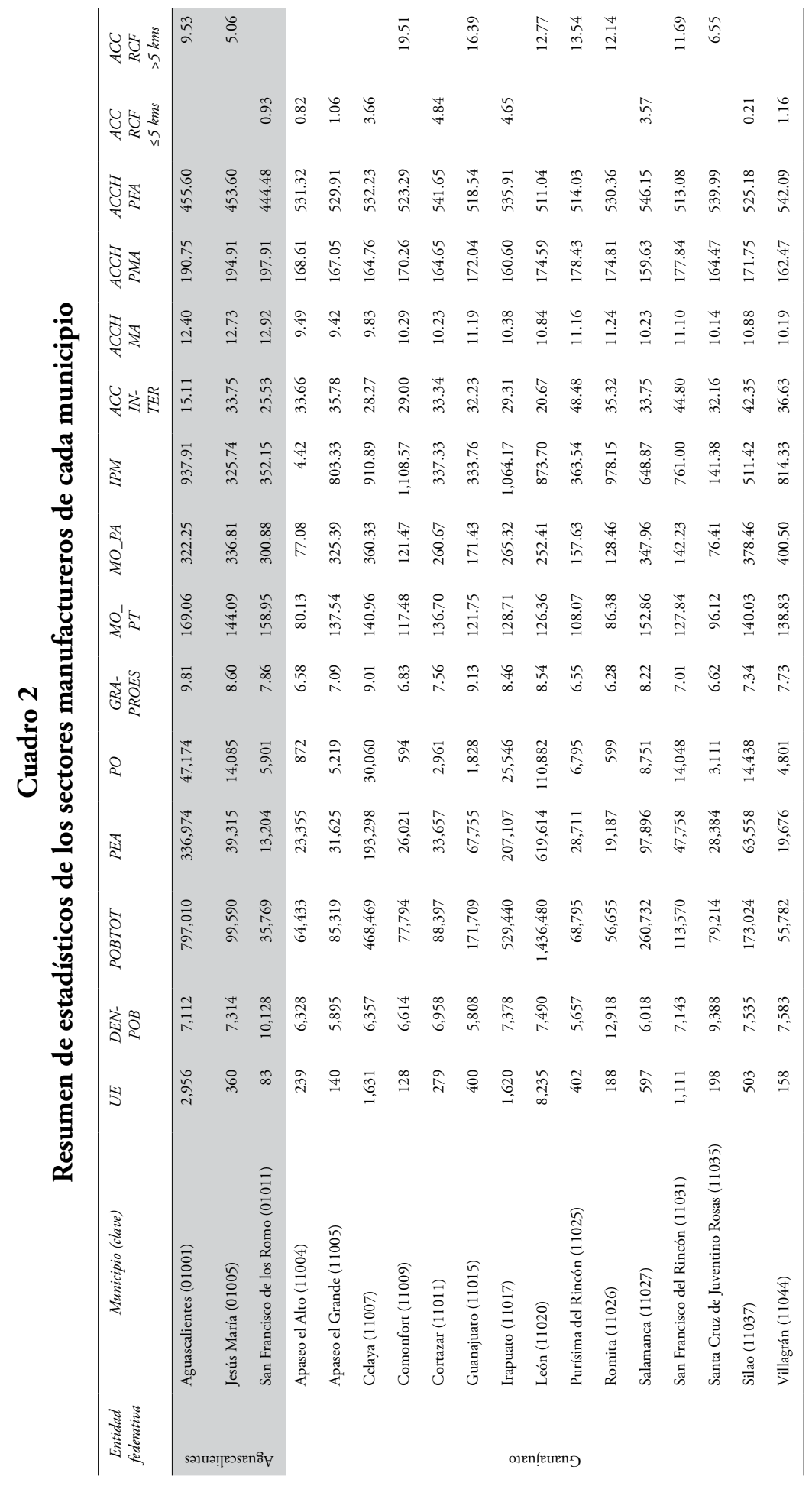




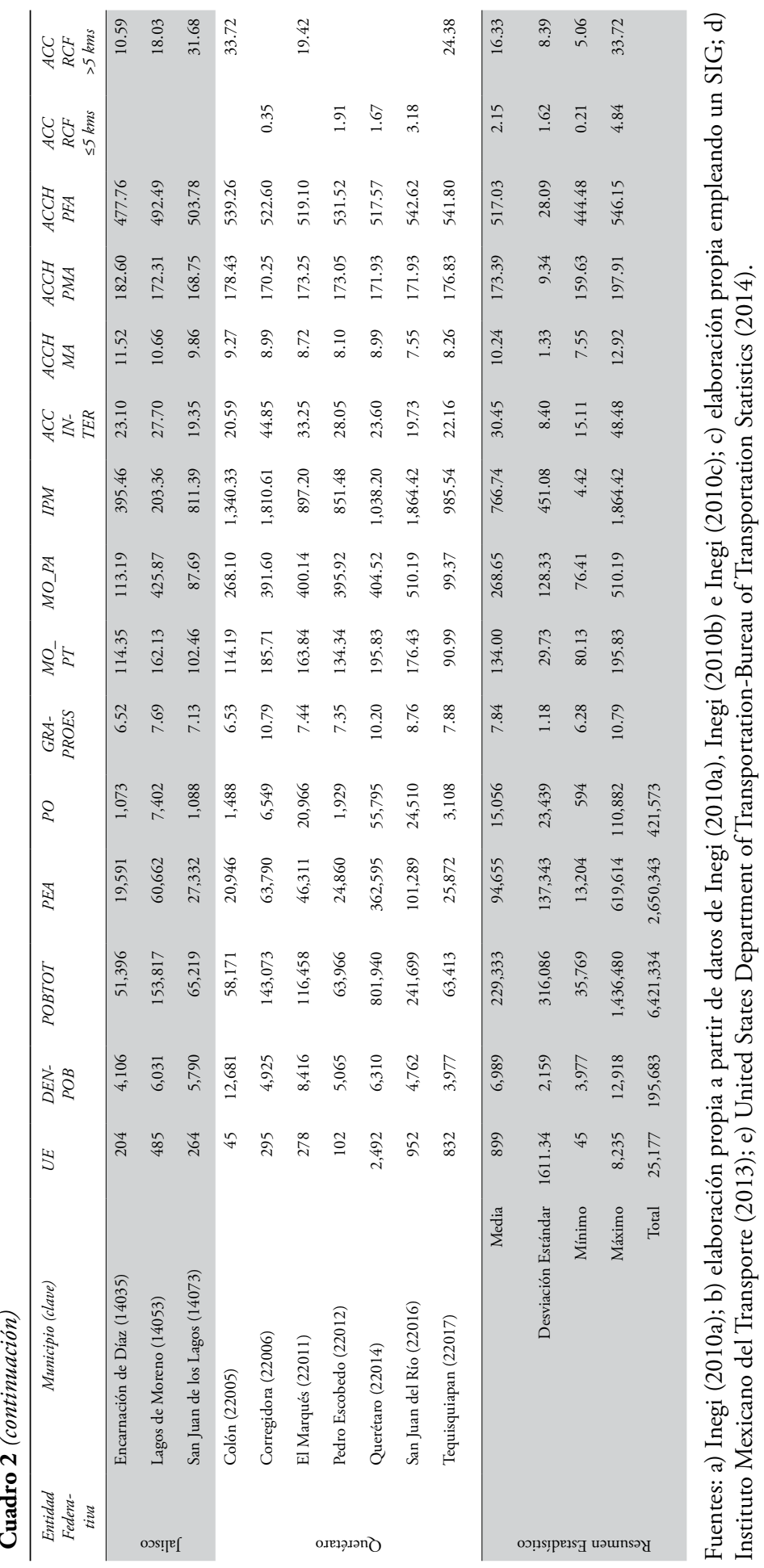




\subsection{La accesibilidad a la demanda interregional}

En la accesibilidad a la demanda se consideran la accesibilidad al mercado interregional y al mercado intrarregional como dos características distintas debido al impacto que se induce en la localización industrial. En Holl (2004a) se expone que la accesibilidad intrarregional debe incrementar los beneficios para una región, dada la conectividad a mercados externos. Sin embargo, la mejora en la accesibilidad interregional aumenta las características económicas de los municipios, por lo que se tienen efectos contrarios al de la demanda intrarregional. En Harris (1954) se indica la función de accesibilidad al mercado (ecuación 4), donde el potencial de demanda para los bienes producidos en el municipio $j$ resulta de la suma del tamaño del mercado del resto de municipios $M_{k}$ dividido entre su distancia $d_{j k}$.

$$
M A_{j}=\sum \frac{M_{k}}{d_{j k}}
$$

En este artículo, la accesibilidad interregional se define como el potencial a la demanda que tienen los municipios hacia la RB, es decir, el mercado interregional sólo incluye la demanda potencial inducida por aquéllos considerados en dicha zona, bajo el supuesto de que tales municipios se comportan como una región económica homogénea. Sin embargo, los beneficios del mercado local dependen del potencial de accesibilidad que recoja cada entidad municipal, es decir, depende directamente del tamaño del mercado.

La distancia $d_{j k}$ es calculada a partir del camino más corto (Dijskstra, 1959), medida en tiempo de viaje, desde el municipio $j$ hacia el resto de municipios de la zona de estudio. Respecto al tamaño del $M_{k}$ no se considera la población tal como proponen Harris (1954), Hanssens et al. (2001), Hall (2004a) y Hall (2004b), pues presenta correlación en la consideración del costo de la renta del suelo debido a que la variable es estimada con la densidad de población, dicha consideración es propuesta en Figueiredo et al. (2002).

Considerando la ecuación 4, se observa que el efecto de la distancia se muestra inversamente proporcional al efecto del tamaño del mercado. Es decir, a mayor tiempo de viaje se incrementa el potencial de accesibilidad. Sin embargo, lo anterior representa mayor costo para el transporte, repercutiendo en una mayor probabilidad de instalación. La consideración de la variable tal como se propone en Holl (2004a) no es acorde con la respuesta esperada en la TUA de McFadden (1974), donde se indica que, dado un conjunto de alternativas, las cuales presentan un vector de atributos que describen las características para cada opción, el individuo preferirá el sitio 
que represente maximizar la utilidad que espera obtener. En este sentido, en Ortúzar y Willumsen (1994) se expone que la probabilidad de que un individuo escoja una cierta opción depende de sus características, y lo atractivo que resulte la alternativa en cuestión, en comparación con las demás.

En este trabajo se propone utilizar el inverso de la formulación de Harris (1954) empleada en Holl (2004a) y Yu et al. (2015), para estimar el potencial de accesibilidad $(A I M)$. Considerando la ecuación 5, a menor tiempo y mayor tamaño del mercado, el valor del potencial disminuye, pero representa mayor accesibilidad a la demanda y menores costos de transporte. Es decir, mayores beneficios; por lo tanto, se espera un signo negativo en el modelo bajo el razonamiento que, a mayor tiempo de viaje, menor probabilidad de que una empresa se instale en determinado lugar.

$$
A I M_{j}=\sum_{k} \frac{d_{j k}}{M_{k}}
$$

\subsection{La accesibilidad a la demanda intrarregional}

Para analizar el efecto que tiene el acceso a la demanda de los mercados intrarregionales, de igual manera que para la demanda interregional, se parte de lo que lo establece Harris (1954), pero se considera el razonamiento de lo esperado en la TUA. En la accesibilidad intrarregional se considera que la función de accesibilidad depende del tamaño del mercado y de la distancia más corta desde cada uno de los municipios de la RB hacia: a) las ocho regiones económicas del país $\left(R_{k}\right)$; b) los principales puertos marítimos $\left(P M_{k}\right)$ y c) los principales puertos de la frontera norte $\left(P F N_{k}\right)$.

Se consideran ocho regiones económicas en el país, las cuales se delimitan en Cordero (1977), y se definen en función de las actividades primarias, secundarias y terciarias, los recursos naturales, el clima, la geomorfología y el territorio. Los puertos marítimos que se consideran en la investigación son los que presentan mayor actividad en el movimiento de contenedores, son dos en la zona del Océano Pacífico: en Manzanillo y en Lázaro Cárdenas, y dos en la zona del Golfo de México: en Veracruz y Altamira (IMT, 2013). Los puertos de la frontera norte se determinan a partir del número de camiones cargados con contenedores que ingresan a los Estados Unidos de América. El puerto con mayor actividad es el de Nuevo Laredo, seguido de Tijuana, Ciudad Juárez y Reynosa (USDOT-BTS, 2014). 
En relación con el tamaño del mercado para las regiones económicas, por un lado Hanssens et al. (2001) indican que los bienes producidos por la industria se venden a los usuarios finales, por lo que asumen que el tamaño del mercado serán personas u hogares. Por otro lado, Harris (1954) expone que una aproximación para estimar el tamaño del mercado es la población, sin embargo, el ingreso familiar o las ventas minoristas proveen un mejor acercamiento. Considerando lo anterior, como medida de aproximación, se propone la Población Económicamente Activa (PEA) bajo el supuesto que es la población de cada entidad federativa, la que conforma cada $R_{k}$, que puede tener el poder económico para adquirir los bienes que se producen.

Por otra parte, debido a la falta de información precisa que describa el tamaño del mercado potencial al que se accede por medio de los puertos de importación-exportación (sean marítimos o terrestres), y siguiendo lo expuesto en Hanssens et al. (2001) -respecto a la idea de estimar el tamaño del mercado considerando que el movimiento eficiente de los bienes producidos requiere canales de distribución desde el centro de producción hasta el mercado final- este acercamiento es considerado una aproximación, hacia los puertos marítimos, el número total de contenedores movilizados es $P M_{k}$, y para los puertos de la frontera norte, el número total de camiones cargados que ingresan a Estados Unidos de América es $P F N_{k}$. Así, más que el tamaño del mercado, se considera como variable la información que refleja la eficiencia que tiene cada puerto para el movimiento de mercancías, pero a su vez está relacionado con los mercados de Estados Unidos de América y del resto de países.

Las ecuaciones 6,7 y 8 definen la función que describe el acceso a la demanda intrarregional para las ocho regiones económicas del país $\left(A R_{j}\right)$, para los principales puertos marítimos $\left(A P M_{j}\right)$, y para los principales puertos de la frontera norte $\left(A P F N_{j}\right)$, respectivamente.

$$
\begin{gathered}
A R_{j}=\sum_{k} \frac{d_{j k}}{R_{k}} \\
A P M_{j}=\sum_{k} \frac{d_{j k}}{P M_{k}} \\
A P F N_{j}=\sum_{k} \frac{d_{j k}}{P M_{k}}
\end{gathered}
$$




\subsection{La accesibilidad a la red carretera federal}

Otra variable a considerar en la localización industrial es la cercanía a la red carretera federal, la cual se analiza en Figueiredo et al. (2002), Holl, (2004a; 2004b), Alañón (2006) y Alañón y Arauzo (2008). Dado que esta cercanía proporciona ventajas en términos de acceso al mercado y a los proveedores, muestra ventajas en costos de transporte, tanto para el suministro de las materias primas como para la distribución del producto final, es entonces una característica que proporciona beneficios a las industrias que deseen instalarse en la región. Los autores anteriormente mencionados no presentan una discusión que sustente la validez del proceso metodológico empleado para el cálculo de la variable que se discute, indican que el acceso a la red carretera federal es el tiempo medio del municipio a la red de carretera de alta capacidad, calculado por medio de Sistemas de Información Geográfica (Alañón, 2006 y Alañón y Arauzo 2008), y el tiempo se calcula considerando una línea recta desde el municipio $j$ a la autopista más cercana (Holl, 2004a y 2004b).

A partir de lo expuesto, se obtiene el centro geométrico de las localidades urbanas correspondientes a cada municipio bajo el supuesto de que la mayor concentración de actividades se encuentra en dicho lugar, por otro lado, es la información espacial más desagregada con la que se dispone para realizar el análisis exploratorio sin violentar la confidencialidad de datos del Inegi.

La conexión para cada municipalidad se efectúa por medio de una línea recta desde el centro geométrico de la localidad urbana hacia la carretera federal más cercana. Para el caso donde el acceso a la red carretera federal tiene dos o más opciones se considera la media de la distancia. Se observan dos estratos en el caso de estudio, el primero, donde el centro geométrico se localiza a menos de $5 \mathrm{~km}$ de distancia a la carretera, en 13 de los 28 municipios de análisis, en los cuales el número de UE representa $36.11 \%$; el segundo, a más de $5 \mathrm{~km}$, donde se localiza $63.89 \%$ de las UE. Por lo anterior, esta variable $(A R C F)$ se considera dicotómica, es decir, para el municipio $j$ donde el centro geográfico se localiza a menos de 5 $\mathrm{km}$, el valor que adopta la variable $k_{\mathrm{j}}$ es igual a 1 , de lo contrario el valor es cero:

Si $A R C F_{j} \leq 5 \mathrm{~km}$, entonces $k_{j}=1$

Caso contrario $=0$ 


\subsection{Los determinantes socioeconómicos de localización}

Se consideran las variables empleadas en Czamanski (1981), Figueiredo et al. (2002), Holl (2004a y 2004b), Alañón (2006), Alañón y Arauzo (2008) y Chin y Hong (2009). Desde los modelos de la teoría clásica de localización (Von Thünen, 1826; Weber, 1929; Christaller, 1933; Lösch; 1940), el costo de la renta del suelo representa una variable determinante para la localización industrial, dadas las ventajas económicas que constituye una porción sobre otra. En Figueiredo et al. (2002) se incluye el costo de la renta del suelo en función de la densidad de población; debido a la falta de información disponible, en esta investigación se adopta lo sugerido por este autor. La densidad de la población se calcula con la población total (Inegi, 2010a) y el área que corresponde a las localidades urbanas de cada municipio que conforma la RB (Inegi, 2010b) (ecuación 9).

$$
D_{\text {ENPOB }}=\frac{\text { POBTOT }_{j}}{\text { ALOC }_{J}}
$$

donde: $D E N P O B_{j}$ es la densidad de población correspondiente al municipio $j, P O B T O T_{j}$ es la población total de las localidades urbanas en el municipio $j$, y $A L O C_{j}$ es el área de las localidades urbanas en el municipio $j$.

El grado promedio de escolaridad señala el nivel de educación que tiene la población, lo cual describe la cualificación de la mano de obra existente en las regiones para analizar, de acuerdo a la TUA, el efecto que se induce al tener acceso a mano de obra calificada en la región (Holl, 2004a; Holl, 2004b; Alañón, 2006 Alañón y Arauzo, 2008) y es obtenida del Inegi (2010a). El tamaño del mercado local se define en función de la PEA, así, considerando la información disponible en el Inegi (2009), permite incluir los costos por la mano de obra desagregados para: a) salarios pagados a obreros y técnicos en producción $\mathrm{C} P T_{i} \mathrm{y} \mathrm{b}$ ) sueldos pagados a empleados administrativos, contables, gerentes y directivos $\mathrm{C} P A_{i}$.

$$
\begin{aligned}
& C P T_{i}=\frac{P T_{i}}{P O T_{j}} \times \frac{1}{365} \\
& C P A_{i}=\frac{P A_{i}}{P O A_{j}} \times \frac{1}{365}
\end{aligned}
$$

El costo de la mano de obra considerado resulta del promedio del gasto anual por el pago que realiza el establecimiento para retribuir el trabajo, es decir, el valor es producto de la división del total del gasto que representa la mano de obra $\left(P T_{i}, P A_{i}\right)$ entre el total de personas que laboraron 
para la empresa en el año 2008 ( $\left.P O T_{i}, P O A\right)$; se divide entre los días del ańo para obtener el costo diario. Lo anterior se aplica para determinar el costo en la ecuación 10 y 11 correspondiente a cada subsector manufacturero (Inegi, 2008). La variable de personal ocupado se obtiene del Inegi (2009).

El indicador de las economías externas marshalianas (cociente de localización o índice de especialización) y el indicador que describe las economías externas derivadas de la diversificación o economías de urbanización (coeficiente de especialización) son variables que se emplean en Holl (2004a y 2004b), y posteriormente se aplican en Alańón (2006) y Alañón y Arauzo (2008). El índice de especialización $C L_{i j}$ representa la relación que existe entre la participación del subsector $i$ en el municipio jy la participación del mismo subsector en el total regional, por lo tanto, se utiliza como medida de la especialización relativa o interregional (ecuación 12). Los rangos de valor que puede adoptar dicho cociente se describen a continuación:

a) Si el tamaño relativo del subsector $i$ en el municipio $j$ es idéntico al tamaño relativo del mismo subsector en la zona de estudio, entonces no existe especialización de la actividad, por lo tanto $C L_{i j}=1$.

b) Si el tamaño relativo del subsector $i$ en el municipio $j$ es menor al tamaño relativo del mismo subsector en la zona de estudio, entonces no existe especialización de la actividad, por lo tanto $C L_{i j}<1$.

c) Si el tamaño relativo del subsector $i$ en el municipio $j$ es mayor al tamaño relativo del mismo subsector de la zona de estudio, entonces se genera una especialización de dicha actividad, por lo tanto, $C L_{i j}>1$.

$$
C L_{i j}=\frac{E_{i j} / E_{j}}{E_{J} / E_{T}}
$$

donde: $E_{i j}$ es el personal ocupado total del subsector manufacturero $i$ en el municipio $j, E_{i}$ el personal ocupado en el municipio $j, E_{J}$ el personal ocupado en la zona de estudio para dicha actividad, y $E_{T}$ el personal ocupado en la zona de estudio en las actividades manufactureras consideradas.

El coeficiente de localización $C E_{i j}$ (ecuación 13) refleja el nivel de similitud de la estructura económica subregional con la estructura económica del patrón de comparación. Se emplea como medida de especialización subregional bajo el supuesto de que la distribución de referencia sea diversificada en términos relativos. De otra manera, el coeficiente refleja el parecido de la estructura de la subregión al ser comparada con la estructura de la región cuando el valor se acerca a cero; mientras el 
valor adopta valores distantes a cero, el parecido tiende a ser nulo. Las variables necesarias para formular el coeficiente de especialización son descritas en el punto anterior.

$$
C E_{i j}=\frac{1}{\sum\left|\left(E_{i j} / E_{l}\right)-\left(E_{J} / E_{T}\right)\right|}
$$

En el mismo sentido a lo expuesto por Mc Fadden (1974), en Ferreira (1995), se indica que mediante la Inversión Pública es posible representar la complementariedad que coexiste entre el capital público y el capital privado, es decir: al incrementar el valor del capital público se desarrolla mayor rentabilidad y productividad para la inversión del capital privado por lo que resulta más atractivo instalarse en los municipios que brinden un mejor y mayor capital público. Lo anterior, debido a que las manufacturas se benefician de la infraestructura pública sin haber invertido directamente en ella. Así, como parámetro de la disponibilidad de cada municipio para captar la inversión del sector manufacturero, se consideran las erogaciones públicas destinadas a la construcción, ampliación, mantenimiento y conservación de las obras públicas mediante información de Inegi (2010c). La variable de capital público considerada en el presente estudio es directamente proporcional al gasto del municipio $j$ más el gasto proveniente del estado $m$ (al que pertenece el municipio $j$ ) e inversamente proporcional a la población total para el municipio $j$.

\section{Resultados y discusión}

Para estimar las características regionales que describen la maximización de beneficios en el sector manufacturero se inicia con la descripción de un modelo que sólo considera las variables económicas y sociales, posteriormente se incluyen una a una las variables que describen la accesibilidad, pues el objetivo es analizar el impacto que induce dicha variable cuando se incluye en el análisis exploratorio. En el cuadro 3 se muestran los modelos estimados.

El modelo 1 incluye las variables: renta del suelo, nivel de educación, población económicamente activa, costo de la mano de obra para el personal de producción, costo de la mano de obra para el personal administrativo, índice de especialización y el coeficiente de localización. El resultado indica que la PEA, el GRAPROES, la MO_PT, el A_INDEX y el SUB_INDEX representan las fuerzas centrípetas, mientras que el resto de variables constituyen las fuerzas centrífugas.

Si se consideran los coeficientes estandarizados (cuadro 4), la PEA resulta el principal incentivo para la instalación manufacturera, es decir, las manufacturas han decidido instalarse en los municipios que presentan 


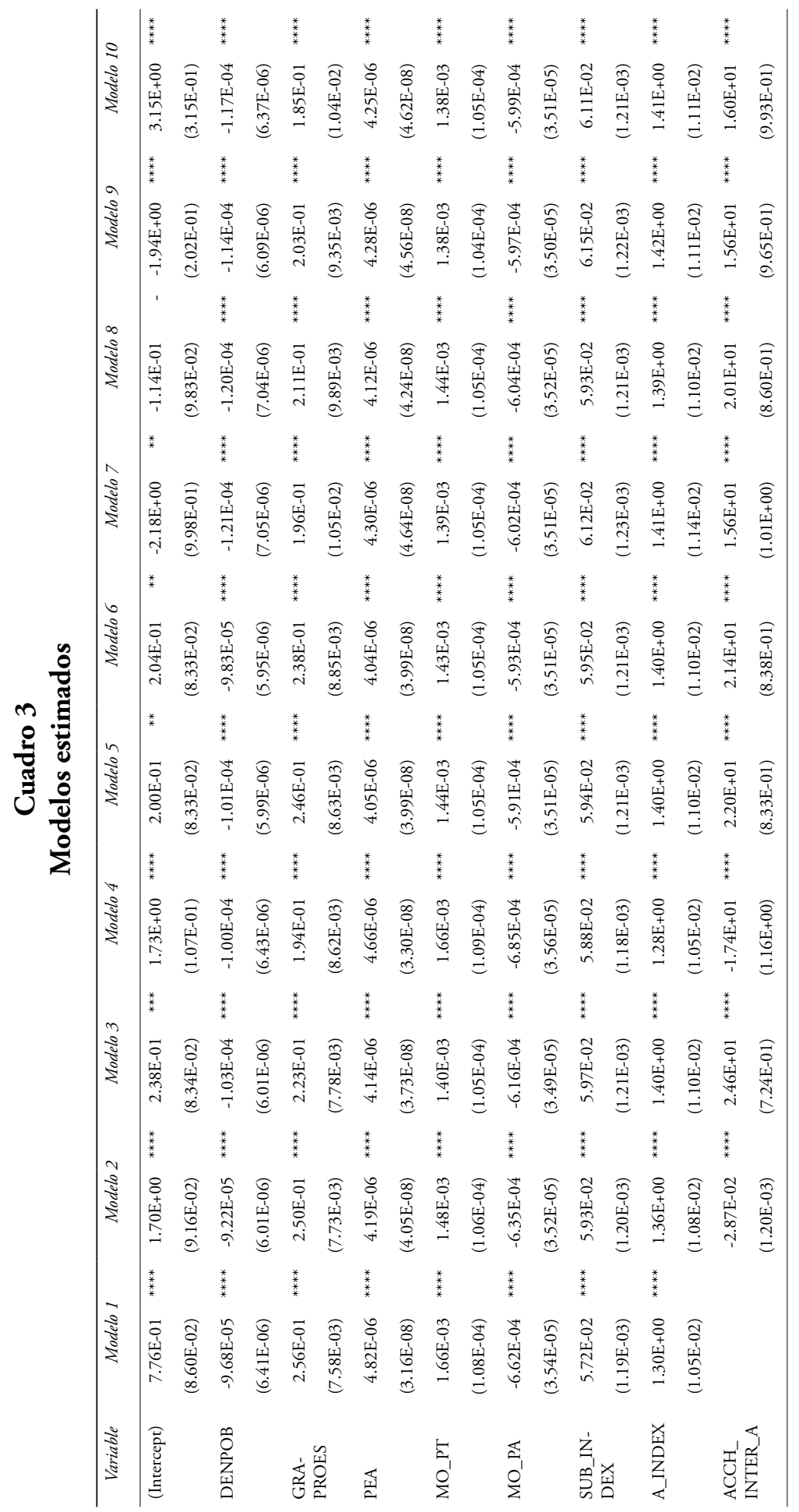




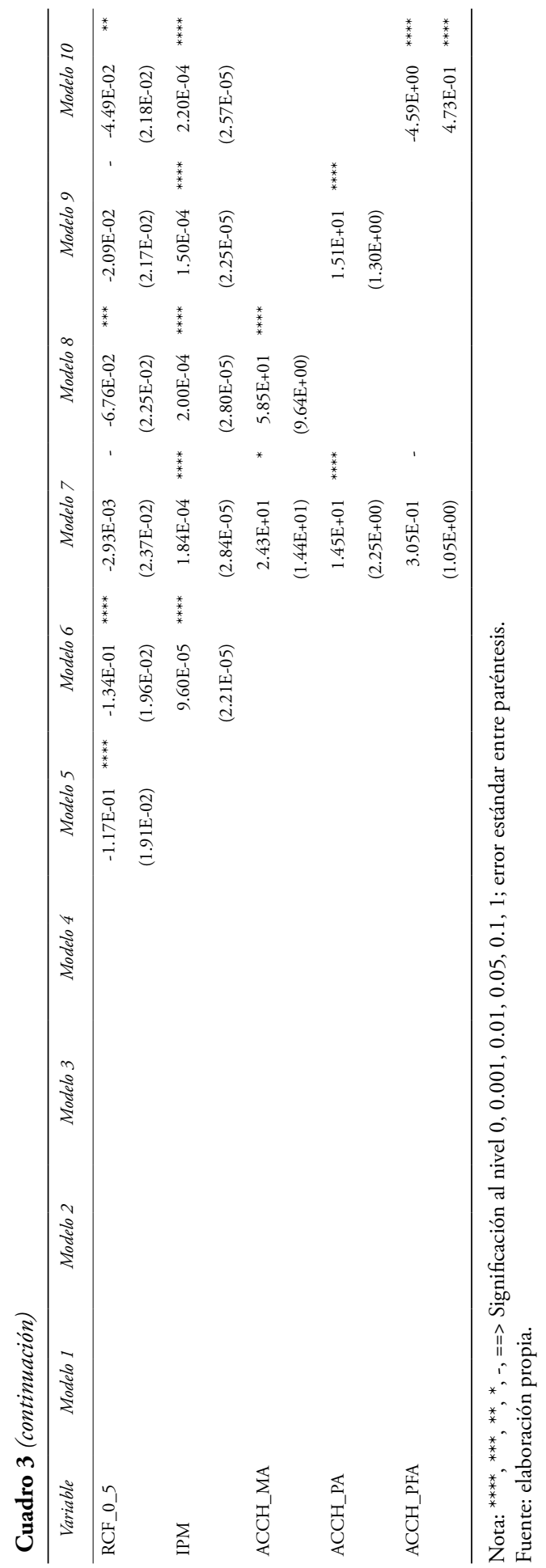




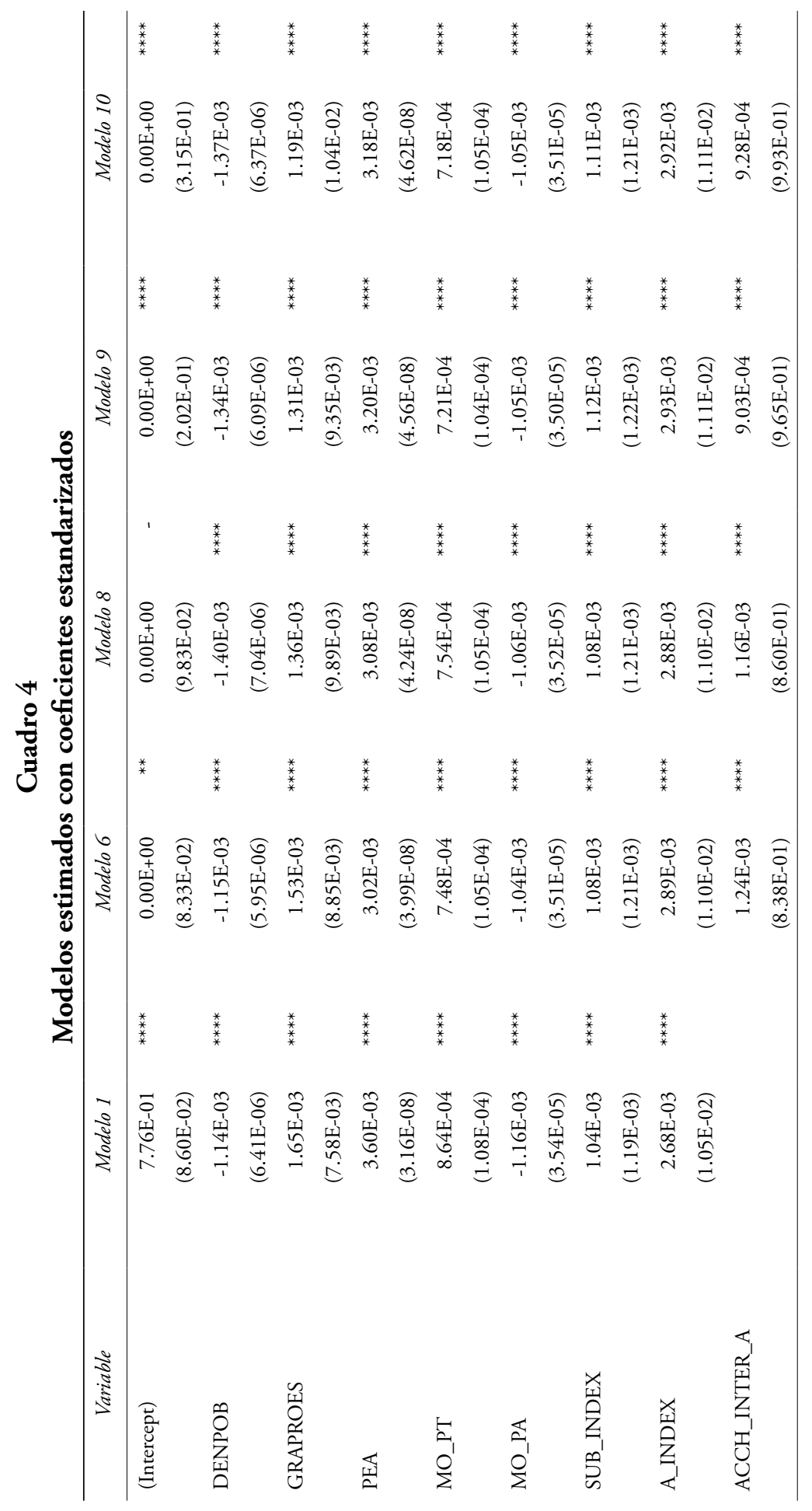




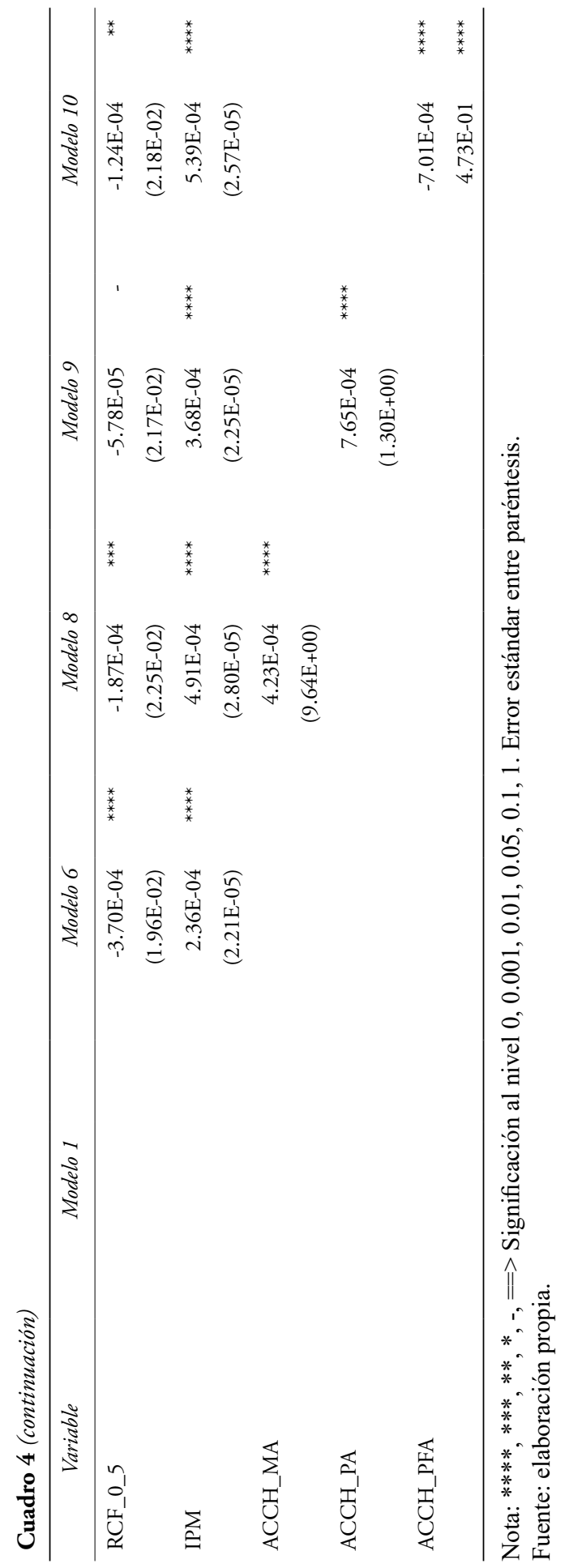


el mayor tamaño del mercado, seguido del GRAPROES, donde es posible reafirmar que conforme se incrementa el nivel educativo, la instalación manufacturera también se presenta en aumento; es decir, se corrobora que la escala de producción se incrementa conforme hay presencia de mano de obra calificada. Lo anterior, en acuerdo a la NGE.

Con menor valor pero altamente significativos el A_INDEX y el SUB_INDEX constituyen incentivos para la localización industrial debido a que la interacción entre empresas, insumos, recursos y personal calificado, estimulan la instalación manufacturera pues los rendimientos se incrementan. Aunado a lo anterior, las economías urbanas propias de grandes aglomeraciones convergen en ventajas para la reducción de costos, derivados de la aglomeración de productores pertenecientes a diversas industrias y de consumidores en un área determinada, lo que genera rendimientos crecientes para las empresas.

En el modelo 1, el principal efecto negativo en la localización industrial lo induce el MO_PA y la DENPOB, es decir, las empresas se han instalado en los municipios donde el costo para la mano de obra, para el personal administrativo y el costo de renta de suelo es mayor. De lo anterior, es posible aseverar que el inversionista prefiere tener acceso a mano de obra calificada aunque su costo sea elevado. Sin embargo, la aproximación para la renta del suelo resulta ambigua pues se observan altos costos de renta de suelo en municipios con menor población que además se encuentran ubicados a la periferia de los principales centros urbanos.

Si se considera la Teoría de los Círculos Concéntricos (Von Thünen, 1826), éstos deberán presentar un costo de renta menor respecto a los principales centros urbanos, pues se localizan a mayor distancia del mercado principal. Lo anterior no ocurre y resulta de la relación que existe entre la extensión territorial de la localidad urbana y la población. El costo de renta de suelo es mayor en ciudades con mayor población respecto a los municipios con menor población, debido a la presencia de los servicios e infraestructura que posee una ciudad en desarrollo.

El modelo 2 incluye variables que se consideran para el modelo 1 más la accesibilidad a la demanda inter-regional, dicha variable refleja signo negativo, indicando que las empresas prefieren instalarse en los municipios que ofrecen menor potencial de accesibilidad hacia el resto de los mercados de la RB, entendiendo que: al incrementar el tiempo de viaje, disminuye el potencial de accesibilidad, lo que resulta en una probabilidad positiva hacia la instalación manufacturera. Sin embargo, incrementar el tiempo de viaje deriva en un mayor costo de transporte.

El modelo 3 incluye el valor inverso para la accesibilidad a la demanda inter-regional (ACCH_INTER_A), es decir, la accesibilidad a la demanda se manifiesta inversamente proporcional al tamańo del mercado 
y directamente proporcional al tiempo de viaje, entendiendo que, a mayor tiempo de viaje, mayores costos de transporte y menor probabilidad de instalación. En la ACCH_INTER_A se exhibe signo positivo, lo que significa que a mayor tiempo de viaje, mayor probabilidad de instalación. Para el caso de estudio, el resultado responde a que la relación de instalación de las empresas entre los municipios con mayor PEA y la mayor actividad manufacturera se ubica geográficamente dispersa entre sí, tal como se observa en el mapa 2, donde los municipios que ofrecen mayor accesibilidad a la demanda regional son aquéllos que se localizan en la periferia de los municipios con el mayor tamańo de mercado, los cuales presentan un tiempo de viaje menor pero también una menor atracción manufacturera.

Para validar los resultados del modelo 3, se estima el modelo 4, el cual parte de una situación hipotética donde se reduce el tiempo de viaje entre los municipios que presentan mayor actividad manufacturera, es decir, dichos municipios se suponen contiguos entre sí, desde el punto de vista territorial. El resto de municipios conservan sus características.

\section{Mapa 2}

\section{Localización geográfica de la industria y su accesibilidad al} mercado regional

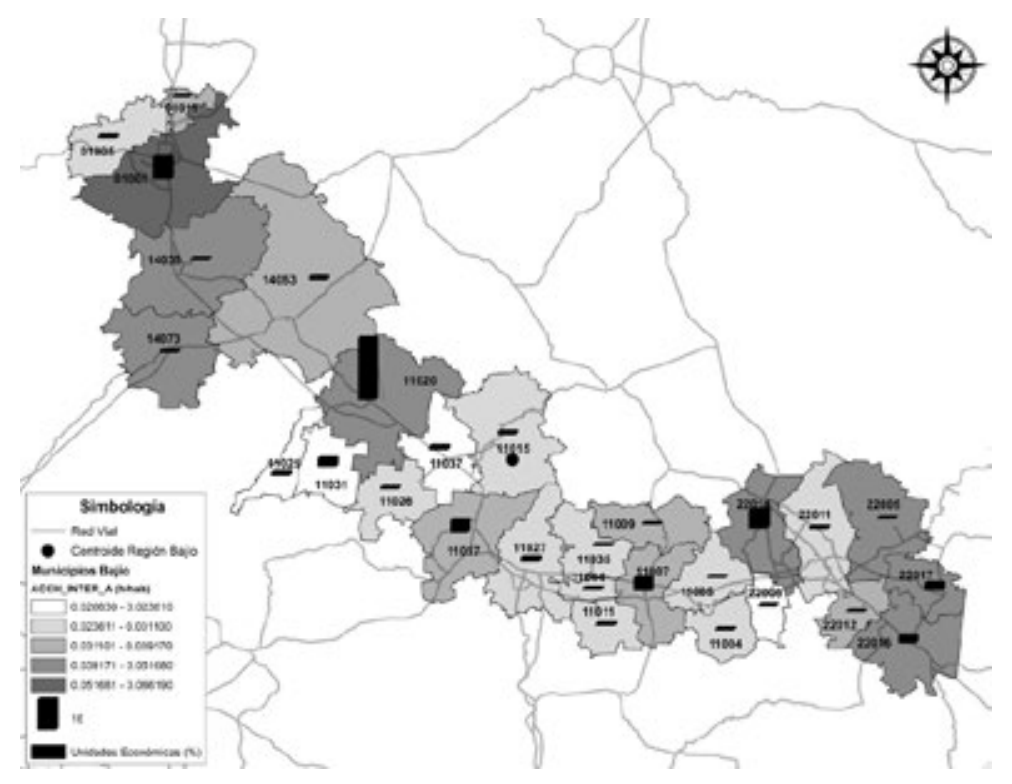

Fuente: elaboración propia. 
En la variable ACCH_INTER_B se refleja dicha situación hipotética y cuando se incluye en el modelo muestra signo negativo, entonces, a mayor tiempo de viaje municipal, menor probabilidad de instalación. El resultado exhibe el efecto que induce la distancia en relación con la conectividad entre regiones, así podemos establecer que la cercanía no es simplemente geográfica sino temporal.

En el modelo 5 se incorporan las variables que se consideran en el modelo 3, más la variable que incluye la accesibilidad a la red carretera federal (RCF_0_5), la cual se considera como valor igual a uno al municipio $j$, que presente una distancia en línea recta desde su centro geográfico a la red carretera federal, menor o igual a cinco kilómetros y cero en caso contrario. En el modelo 5 se refleja que la variable RCF_0_5 obtiene signo positivo, mostrando que la industria se instala a más de $5 \mathrm{~km}$ de distancia de la red carretera federal.

El número de empresas se incrementa conforme crecen los costos de transporte, por lo que el signo resultante es inverso al signo esperado. El resultado indica que el método que se propone en Figueiredo et al. (2002), Holl (2004a y 2004b), Alañón (2006) y Alañón y Arauzo (2008) para determinar la distancia a la red carretera presenta una desventaja, dado que se considera el centro geométrico del municipio $j$ y no la ubicación real de las empresas manufactureras, o en su defecto de los parques o zonas industriales, pues su ubicación principalmente se presenta adyacente a la red viaria federal como se corrobora en Obregón-Biosca et al. (2016). Por lo anterior, se propone para futuros análisis obtener para cada municipio la media de la distancia de cada parque industrial o empresa a la red carretera federal, similar al que se emplea en Obregón-Biosca et al. (2014) hacia puertos de exportación.

En el modelo 6 se agrega la variable que considera el egreso estatal y municipal para la Inversión Pública (IPM), dicha variable adquiere signo positivo, lo que se interpreta como un incentivo en la instalación manufacturera. El valor del capital público refleja una mejora para el patrimonio de cada municipio, es por lo anterior que el resultado muestra una relación positiva y acorde a la TUA respecto al incremento en la actividad manufacturera. El resultado exhibe que las regiones donde se muestra un mayor desarrollo económico son aquéllas donde se presenta mayor inversión en infraestructura, lo cual es acorde a lo que se expone en Berechman (1994).

En el modelo 7 se incluye la accesibilidad intra-regional hacia cada una de las ocho regiones económicas de México (ACCH_MA), a los puertos marítimos (ACCH_PA) y a los puertos de la frontera norte (ACCH_PFA), mientras que los modelos 8, 9 y 10 incluyen la $\mathrm{ACCH}$ $\mathrm{MA}$, la ACCH_PA y la ACCH_PFA, respectivamente. Dichas variables 
se incluyen tomando como referencia el modelo 6. En los resultados se expresa que al incluir la RCF_0_5, la ACCH_MA, y la ACCH_PFA en un solo modelo, éstas no son estadísticamente representativas.

El resultado precedente se infiere al analizar la influencia de la $\mathrm{ACCH}_{-}$ MA sobre la ACCH_PFA, la que se presenta como inversa, es decir, mientras que el potencial de accesibilidad a la demanda de los mercados nacionales ofrece rendimientos crecientes, la accesibilidad a los puertos de la frontera norte decrece los beneficios esperados. Por lo anterior, se considera cada variable en un modelo independiente. Además, se tiene por objetivo analizar el efecto que induce la accesibilidad hacia los principales mercados de México y hacia los principales puertos de importaciónexportación.

$\mathrm{Al}$ contrastar los resultados del modelo 6 con los resultados obtenidos en los modelos 8, 9 y 10 se tiene que: a) en el modelo 9, la RCF_0_5 no es estadísticamente representativa y la ACCH_PA refleja signo positivo; b) en el modelo 10, con la ACCH_INTER_A se refleja un cambio de signo; c) en el modelo 8, la ACCH_MA presenta signo positivo.

A partir de lo expuesto, se infiere que las unidades económicas se localizan en los municipios que ofrecen mayor costo de transporte para acceder a los puertos marítimos de importación-exportación. La ACCH_ PFA que se incluye en el modelo 10 muestra el efecto inverso respecto a la ACCH_MA y la ACCH_PA que se consideran en los modelos 8 y 9, respectivamente. Se muestra que el signo del coeficiente para la demanda a la accesibilidad a los puertos fronterizos es negativo; el resultado se debe a que las empresas se localizan en los municipios que brindan mayor acceso a la región norte del país. El tiempo de viaje hacia los puertos fronterizos induce un impacto significativo en el potencial de accesibilidad. En otras palabras, incrementar la distancia a los puertos fronterizos implicará menor probabilidad de instalación manufacturera. Los resultados descritos con anterioridad son acordes a la TUA.

Con la finalidad de discutir las variables que se destacan en la región en la instalación manufacturera, en el cuadro 4 se muestran los modelos más representativos y sus coeficientes estandarizados para cada variable independiente. La estandarización de coeficientes permite comparar las variables independientes al homogeneizar la escala de medida en unidades de desviación estándar (Nathans et al., 2012). Dado que el proceso para determinar el coeficiente estandarizado considera la contribución de todas las variables incluidas en el modelo de regresión, cada valor del coeficiente estandarizado para la variable independiente es un valor del efecto total que ejerce a la variable dependiente (Lebreton et al., 2004).

En el modelo 1 se refleja que la PEA resulta ser el principal incentivo para la instalación manufacturera, seguido del A_INDEX y el GRA- 
PROES. Cuando se incluye en el modelo 6 la IPM, la ACC_INTER y la ACC_RCF, la PEA continúa siendo la variable que mayor peso presenta en el modelo, seguida del A_INDEX y del GRAPROES, mientras que la IPM es la cuarta variable con mayor peso. Para el modelo 8, en el cual se incluye el potencial de accesibilidad a la demanda de las ocho regiones económicas del país, la variable que presenta la mayor contribución a la variable independiente es la PEA, seguida del A_INDEX, la DENPOB y el GRAPROES. El modelo 9 incluye el potencial de accesibilidad a los principales puertos marítimos, y el modelo 10 el potencial de accesibilidad a los principales puertos de la frontera norte; las cuatro variables que presentan mayor contribución a la variable independiente son las mismas y en igual orden que en el modelo 8. Considerando el modelo 8, el coeficiente de la PEA es 1.07 veces superior al A_INDEX (3.08/2.88), 2.19 veces respecto a la DENPOB, de 2.27 respecto al GRAPROES y 6.27 veces respecto a la IPM.

El análisis de los coeficientes estandarizados de las variables independientes determina que la PEA es la característica que más se destaca en territorio, en el sentido de ofrecer mayores beneficios en la instalación manufacturera. La industria se ha visto beneficiada por las economías urbanas inducidas por la diversidad de subsectores instalados en la zona de estudio, mientras que la DENPOB muestra ser la tercer característica que proporciona mayores beneficios, seguida del GRAPROES. Aunque las variables que describen la accesibilidad se manifiestan como significativas, tras el análisis de los coeficientes estandarizados, resultan con una contribución menor que el resto de variables, es decir, los beneficios obtenidos o esperados que son inducidos por los costos de transporte, no son el principal atractivo para la instalación manufacturera en la RB.

\section{Conclusiones}

De acuerdo a las condiciones establecidas en esta investigación, la teoría que se aborda, la metodología que se expone, los resultados que se obtienen y el análisis de los mismos, la accesibilidad inducida por la infraestructura del transporte en el mercado regional, hacia el mercado nacional y los puertos de importación-exportación no resulta ser la característica con mayor significación en comparación al resto de variables que aproximan a explicar la instalación manufacturera en la Región Bajío. Se comprueba que la infraestructura de transporte es un factor necesario pero no suficiente en el emplazamiento industrial.

En los resultados se muestra que las variables que describen el potencial de accesibilidad a la demanda (interregional e intrarregional) exhiben una aproximación altamente significativa según el resultado que refleja la 
prueba de bondad de ajuste y chi-cuadrada. Dichas variables explican la localización manufacturera en la zona de estudio en conjunto con las características restantes.

Las variables donde se muestra mayor significancia para describir el número de empresas localizadas en la Región Bajío en orden de importancia, a partir de los coeficientes estandarizados son: a) el tamaño del mercado local; b) las economías de urbanización; c) el costo de renta del suelo y d) el grado promedio de escolaridad.

Cuando se analiza el efecto que induce el tiempo de viaje en el potencial de accesibilidad que se propone en Harris (1954), se observa que la aproximación más sensible es considerar el potencial como una función directamente proporcional al tiempo de viaje e inversamente proporcional al mercado. Lo anterior se infiere al contraponer las variables y observar el comportamiento que se presenta. Si se considera el tamaño del mercado como la PEA y la distancia como tiempo de viaje, el valor para el tiempo de viaje es una aproximación estrecha al costo de transporte debido a la influencia que representa el tiempo de traslado desde el centro de producción hasta el mercado potencial.

Caso contrario, en la PEA se considera una aproximación más cercana al tamaño del mercado con respecto a la población total. No obstante, resulta ambiguo definir a la PEA como el tamaño del mercado debido a que dicha población no precisa la necesidad de adquirir los bienes producidos por la industria manufacturera y menos la población total, tal como se considera en Harris (1954) y se emplea en Holl, 2004a, y recientemente en Yu et al. (2015).

Con relación a lo que se describe en los párrafos precedentes, en esta investigación se destaca la consideración de la PEA como el estrato de la población que define el tamaño del mercado, un estrato que selecciona a la población que trabaja o trabajó y percibe un salario. Con ello, es viable aproximar a dicha población como el mercado potencial, es decir, la población que tiene poder económico de compra. A partir de la información disponible se permite desagregar el costo de la mano de obra para el personal técnico de producción y el personal administrativo, dando pie a un análisis con niveles de menor agregación respecto a los que se realizan en Holl (2004a y 2004b); Alañón (2006); Alañón y Arauzo (2008), Obregón-Biosca et al. (2014) y Yu et al. (2015).

Desde el punto de vista neoclásico, el modelo lineal generalizado que se implementa en esta investigación para explicar el número de empresas que se instalan en determinada región, dadas sus características económicas (Teoría de Utilidad Aleatoria), resultó ser una herramienta útil de acuerdo con lo que se establece en Guimaráes et al. (2003) y los resultados de Holl (2004a y 2004b). Sobresale la ventaja de utilizar el modelo de 
regresión de Poisson para determinar las características del territorio utilizando datos a nivel geográfico local. Se propone como línea de investigación futura abordar un análisis, que incluya parámetros de la escuela institucional y neoclásica, a nivel desagregado (por empresa), para determinar qué características influyen en la instalación manufacturera por subsector manufacturero en una o más regiones y contrastarlas a través del tiempo mediante datos de panel.

\section{Fuentes consultadas}

Alañón, Ángel y Josep María Arauzo (2008), “Accesibilidad y localización industrial. Una aplicación a las regiones españolas fronterizas con Francia", Revista de Estudios Regionales, núm. 82, Universidades Públicas de Andalucía, Málaga, España, pp. 71-103.

Alañón, Ángel (2006), "Análisis espacial de la creación de establecimientos manufactureros en los municipios andaluces", Revista de estudios regionales, núm. 76, Universidades Públicas de Andalucía, Málaga, España, pp. 135-159.

Alañón, Ángel (2002), “Estimación del valor añadido per cápita de los municipios españoles en 1991 mediante técnicas de econometría espacial”, Ekonomiaz, núm. 51, Gobierno Vasco, San Sebastián, España, pp. 172-194.

Banister, David y Joseph Berechman (2001), "Transport investment and the promotion of economic growth", Journal of Transport Geography, 9 (3), Elsevier B.V., Ámsterdam, Países Bajos, pp. 209-218.

Bassols-Batalla, Ángel (1993), Formación de regiones económicas, Universidad Nacional Autónoma de México, Ciudad de México, México.

Bassols-Batalla, Ángel (1979), Geografia, subdesarrollo y regionalización, Editorial Nuestro Tiempo, Ciudad de México, México.

Beckmann, Martin (1999), Lectures on location theory, Springer, Heidelberg, Alemania.

Berechman, Joseph (1994), "Urban and regional economic impacts of transportation investment: a critical assessment and proposed methodology", Transportation research part A: policy and practice, 28 (4), Elsevier B.V., Ámsterdam, Países Bajos, pp. 351-362. 
Chasco-Yrigoyen, Coro y Ana López-García (2010), "Evolution of the influence of geography on the location of production in Spain", en Antonio Páez, Ron Buliung, Julie Le Gallo y Sandy Dall'erba (eds.), Progress in spatial analysis: methods and applications, Springer-Verlag Berlin Heidelberg, Heidelberg, Alemania, pp. 407-440.

Chin, Antoni y Junjuie Hong (2009), “The location decisions of foreign logistics firms in China: does transport network capacity matter?", en Peter Wilson (ed.), Economic Policies and Social Welfare in the 21st Century: challenges and responses for China and Thailand, Cengage Learning Asia Pte Ltd, , Singapur, pp. 169-191

Christaller, Walter (1933) (1966), Die zentralen Orte in Süddeutschland (Central places in southern Germany), traducción de Charlisle Baskin, Prentice Hall, Nueva York, Estado Unidos de América.

Cohen, Jeffrey y Catherine Paul (2005), “Agglomeration economies and industry location decisions: the impacts of spatial and industrial spillovers", Regional Science and Urban Economics, 35 (3), Elsevier B.V., Ámsterdam, Países Bajos, pp. 215-237.

Cordero, Salvador (1977), Concentración industrial y poder económico en México, El Colegio de México, Ciudad de México, México.

Czamanski, Daniel (1981), "Some considerations concerning industrial location decisions", European Journal of Operations Research, 6 (2), Elsevier B.V., Ámsterdam, Países Bajos, pp. 227-231.

Dijkstra, Edsger (1959), "A note on two problems in connexion with graph", Numerische Mathematik, 1 (1), Springer, Heidelberg, Alemania, pp. 269-271.

Ferreira, Francisco (1995), "Roads to equality: wealth distribution dynamics with public-private capital complementarity", reporte técnico núm. TE, 286, Suntory and Toyota International Centres for Economics and Related Disciplines, London School of Economics and Political Science, Londres, Inglaterra.

Figueiredo, Octávio, Paulo Guimarães y Douglas Woodward (2002), "Home-field advantage: location decisions of Portuguese entre- 
preneurs", Journal of Urban Economics, 52 (2), Elsevier B.V., Ámsterdam, Países Bajos, pp. 341-361.

Fujita, Masahisa, Paul Krugman y Antony Venables (1999), The spatial economy, cities, regions and international trade, The MIT Press, Massachusetts, Estados Unidos de América.

Fujita, Masahisa y Jaques Thisse (1996), "Economics of agglomerations", Journal of the japanese and international economies, 10 (4), Elsevier B.V., Ámsterdam, Países Bajos, pp. 339-378.

Guimarães, Paulo, Octávio Figueiredo y Douglas Woodward (2003), “A tractable approach to the firm location decision", The Review of Economics and Statistics, 85 (1), The MIT Press, Massachusetts, Estados Unidos de América, pp. 201-204.

Hanssens, Dominique, Leonard Parsons y Randall Schultz (2001), Market response models: econometric and time series analysis, Kluwer Academic Publishers, Massachusetts, Estados Unidos de América.

Harris, Chauncy (1954), "The market as a factor in the localization of industry in the United States", Annals of the Association of American Geographers, 44 (4), Taylor \& Francis, Londres, Inglaterra, pp. 315-348.

Hayter, Roger (1997), The dynamics of industrial location. The Factory, the firm and the production system, Wiley \& Sons Ltd., Nueva York, Estados Unidos de América.

Holl, Aldelheid (2004a), "Manufacturing location and impacts of road transport infrastructure: empirical evidence from Spain”, Regional Science and Urban Economics, 34 (3), Elsevier B.V., Ámsterdam, Países Bajos, pp. 341-363.

Holl, Aldelheid (2004b), "Transport infrastructure, agglomeration economies, and firm birth: empirical evidence from Portugal", Journal of Regional Science, 44 (4), Wiley, Nueva Jersey, Estados Unidos de América, pp. 693-712.

Hoover, Edgar y Frank Giarratani (1971), An introduction to Regional Economics, McGraw-Hill, Nueva York, Estados Unidos de América. 
IMT (Instituto Mexicano del Transporte) (2013), Manual estadístico del sector transporte 2013, Secretaría de Comunicaciones y Transportes-Instituto Mexicano del Transporte, Querétaro, México.

Inegi (Instituto Nacional de Estadística y Geografía) (2008), "Sistema de Clasificación Industrial de América del Norte, México SCIAN 2007”, Inegi, Aguascalientes, México.

Inegi (Instituto Nacional de Estadística y Geografía) (2009), “Censos Económicos 2009”, Inegi, Aguascalientes, México, <http:// www3.inegi.org.mx/sistemas/saic/>, 23 de marzo de 2015.

Inegi (Instituto Nacional de Estadística y Geografía) (2010a), “Censo de Población y Vivienda 2010”, Inegi, Aguascalientes, México, <http://www.inegi.org.mx/sistemas/consulta_resultados/ iter2010.aspx? $\mathrm{c}=27329 \&$ s=est $>, 15$ de abril de 2015 .

Inegi (Instituto Nacional de Estadística y Geografía) (2010b), “Cartografía geoestadística urbana, cierre del Censo de Población y Vivienda 2010", Inegi, Aguascalientes, México, <http://www.inegi.org. $\mathrm{mx} / \mathrm{geo} /$ contenidos/urbana/default.aspx>, 13 de abril de 2015 .

Inegi (Instituto Nacional de Estadística y Geografía) (2010c), "Finanzas públicas estatales y municipales 2010”, Inegi, Aguascalientes, México, <http://www.inegi.org.mx/est/contenidos/proyectos/registros/economicas/finanzas/default.aspx>, 17 de marzo de 2015.

Johansson, Börje y John Quigley (2004), “Agglomeration and networks in spatial economies" en Raymond Florax y David Plane (eds.), Fifty years of Regional Science, Springer, Berlin Heidelberg, Heidelberg, Alemania, pp. 165-176.

Krugman, Paul (1998), Geografía y comercio, Antoni Bosch, Barcelona, España.

Lebreton, James, Robert Ployhart y Robert Ladd (2004), “A Monte Carlo comparison of relative importance methodologies", Organizational Research Methods, 7 (3), Sage Publications Inc., Los Ángeles, Estados Unidos de América, pp. 258-282.

Lösch, August (1940), The economics of location, Yale University Press, New Haven-Londres, Inglaterra. 
Marshall, Alfred (1920), Principles of Economics, Macmillan, Londres, Inglaterra.

McFadden, Daniel (1974), "Conditional logit analysis of qualitative choice behavior", en Paul Zarembka (ed.), Frontiers in Econometrics, Academic Press, Nueva York, Estados Unidos de América, pp. 105-142.

Mendoza-Cota, Jorge y Jorge Pérez-Cruz (2007), “Aglomeración, encadenamientos industriales y cambios de localización manufacturera en México", Economía, Sociedad y Territorio, VI (23), El Colegio Mexiquense, A. C., Toluca, México, pp. 655-691.

Nathans, Laura, Frederick Oswald y Kim Nimon (2012), "Interpreting multiple linear regressions: a guidebook of variable importance", Practical Assessment, Research \& Evaluation, 17 (9), PAREonline, Estados Unidos de América, pp. 1-19.

Obregón-Biosca, Saúl Antonio, José Antonio Romero-Navarrete, Juan Fernando Mendoza-Sánchez y Eduardo Betanzo-Quezada, (2016), "Impact of mobility induced by urban sprawl: case study of the Querétaro Metropolitan Area", Journal of Urban Planning and Development, 142 (2), American Society of Civil Engineers, Virginia, Estados Unidos de América, pp. 1-12, doi: 10.1061/ (ASCE)UP.1943-5444.0000295.

Obregón-Biosca, Saúl Antonio, José Manuel Chávez-Usla y Eduardo Betanzo-Quezada (2014), "Road transport infrastructure and manufacturing location: an empirical evidence and comparative study between Tijuana and Nuevo Laredo, Mexico", Frontera Norte, 26 (52), El Colegio de la Frontera Norte, Mexicali, Baja California, México, pp. 109-133.

Obregón-Biosca, Saúl Antonio y Rosa Junyent-Comas (2011), "The socioeconomic impact of the roads: a case of study of the 'Eix Transversal' in Catalonia, Spain", Journal of Urban Planning and Development, 137 (2), American Society of Civil Engineers, Virginia, Estados Unidos de América, pp. 159-170.

Obregón-Biosca, Saúl Antonio (2010), "Estudio comparativo del impacto en el desarrollo socioeconómico en dos carreteras: Eix Transversal de Cataluña, España y MEX120, México", Economía, So- 
ciedady Territorio, X (32), El Colegio Mexiquense, A. C., Toluca, México, pp. 1-47.

Ortúzar, Juan de Dios y Luis Willumsen (1994), Modelling transport, John Wiley \& Sons Ltd., Chichester, Reino Unido.

Rodríguez-González, Juan y Alex Caldera-Ortega (2013), "Crecimiento económico y desarrollo local en la región Centro-Bajío de México", Quivera, 15 (1), Universidad Autónoma del Estado de México, Toluca, México, pp. 35-57.

Sánchez-Reaza, Javier (2010), “Trade, proximity and growth: the impact of economic integration on Mexico's regional disparities", Integration \& Trade Journal, 14 (31), Inter-American Development Bank, Washington, Estados Unidos de América, pp. 19-32.

Sedesol-Conapo-Inegi (Secretaría de Desarrollo Social-Consejo Nacional de Población-Instituto Nacional de Estadística y Geografía) (2012), Delimitación de las Zonas Metropolitanas de México 2010, Sedesol-Conapo-Inegi, Ciudad de México, México

Small, Kenneth (1982), Geographically differentiated taxes and the location of firms, Transaction Publishers, New Jersey, Estados Unidos de América.

Unger, Kurt, José Ibarra y Rafael Garduño (2013), “Especializaciones reveladas y ventajas competitivas en el bajío mexicano", documento de Trabajo E-550, Centro de Investigación y Docencia Económicas, Ciudad de México, México.

USDOT-BTS (U.S. Department of Transportation - Bureau of Transportation Statistics) (2014), "Border Crossing/Entry Data", USDOTBTS, Washington, Estados Unidos de América, <https://transborder.bts.gov/programs/international/transborder/TBDR_BC/ TBDR_BC_Index.html>, 24 de noviembre de 2014.

Von Thünen, Johann (1826) (1966), Der isolierte Staat, The Isolated State, Pergamon Press, Oxford, Inglaterra.

Weber, Alfred (1929) (1909), Theory of the location of industries, University of Chicago Press, Chicago, Estados Unidos de América. 
Yu, Nannan, Gert Roo, Martin Jong y Servaas Storm (2015), "Does the expansión of a motorway network lead to economic agglomeration? Evidence from China", Transport Policy, núm. 45, Elsevier B.V., Ámsterdam, Países Bajos, pp. 218-227, doi: 10.1016/j. tranpol.2015.03.014.

Recibido: 29 de octubre de 2015. Corregido: 30 de enero de 2016. Aceptado: 28 de enero de 2017.

Gerardo Ríos-Quezada. Maestro en Ingeniería de vías terrestres por la Universidad Autónoma de Querétaro. Actualmente es investigador del Instituto Mexicano del Transporte en la Coordinación de Seguridad y Operación del Transporte. Su línea de investigación actual se centra en el análisis del ordenamiento territorial. Entre sus últimas publicaciones destacan: "Industrial location and national transport infrastructure: an empirical evidence between Queretaro and Tijuana Metropolitan Zones, Mexico", en Albert Tavidze (ed.), Progress in Economics Research, vol 33, Nova Science Publishers, Nueva York, Estado Unidos de América, pp. 175-196 (2015).

Saúl Antonio Obregón-Biosca. Doctor en ingeniería por el Departamento de Infraestructuras del Transporte y Ordenación del Territorio de la Universidad Politécnica de Cataluña. Actualmente es profesor-investigador en la División de Estudios de Posgrado de la Facultad de Ingeniería de la Universidad Autónoma de Querétaro, donde coordina la maestría en Vías terrestres y movilidad. Es miembro del Sistema Nacional de Investigadores, nivel I. Su línea de investigación actual se centra en el análisis de movilidad metropolitana y externalidades del transporte. Entre sus últimas publicaciones como autor destacan: "Road transport infrastructure and manufacturing location: an empirical evidence and comparative study between Tijuana and Nuevo Laredo, Mexico", Frontera Norte, 26 (52), El Colegio de la Frontera Norte, Tijuana, Baja California, México, pp. 109-132 (2014); "Análisis de la movilidad urbana de una ciudad media mexicana, caso de estudio: Santiago de Querétaro", Economí, Sociedady Territorio, XV (47), El Colegio Mexiquense, A. C., Toluca, México, pp. 61-98 (2015); "Industrial location and national transport infrastructure: an empirical evidence between Queretaro and Tijuana Metropolitan Zones, Mexico", en Albert Tavidze (ed.), Progress in Economics Research, vol. 33, Nova Science Publishers, Nueva York, Estados 
Unidos de América, pp. 175-196 (2015); "Impact of mobility induced by urban sprawl: case study of the Querétaro Metropolitan Area", Journal of Urban Planning and Development, American Society of Civil Engineers, 142 (2), American Society of Civil Engineers, Virginia, Estados Unidos de América, pp. 1-12, doi: 10.1061/(ASCE)UP.1943-5444.0000295 (2015); "Dispersión urbana e integración funcional al núcleo central. Caso de estudio: Zona Metropolitana de Querétaro, México", Revista Gestión y Política Pública, XXIV (2), Centro de Investigación y Docencia Económicas A. C., Ciudad de México, México, pp. $491-531$ (2015). 